\title{
Structural characterization of a novel amino acid decarboxylase
}

Raquel S. Cordeiro ${ }^{1,2}$, Maria Håkansson² ${ }^{2}$ Derek Logan², Björn Walse ${ }^{2}$ and Robert Kourist ${ }^{3}$

\author{
${ }^{1}$ Junior Research Group for Microbial Biotechnology, Ruhr-University Bochum, \\ Universitätstr. 150, 44780 Bochum, Germany \\ ${ }^{2}$ SARomics Biostructures AB, Medicon Village, SE-223 81 Lund, Sweden \\ ${ }^{3}$ Institute of Molecular Biotechnology - TU Graz, Petersgasse 148010 Graz, Austria \\ Corresponding author: Raquel.CorreiaCordeiro@rub.de
}

The introduction of new enzyme activities by protein engineering allows insights into the molecular determinants of catalysis and how new enzymatic functions might have emerged. It also provides leads for the generation of enzymes for new and unnatural reactions ${ }^{(1)}$. Amino acid decarboxylases (AAD) are usually very specific for their natural substrates. For the production of optically pure amines from branched-chain amino acids, we focus on a lysine decarboxylase (LDC) that was recently evolved from a 2,4-diaminobutyrate decarboxylase family, thus presumably having a wider substrate spectrum and higher plasticity for enzyme engineering ${ }^{(2)}$.

The novel AAD which belongs to the pyridoxal-5'-phosphate (PLP)-dependent enzymes was successfully crystallized and it diffracted at $2.5 \AA$ resolution. The X-ray structure was solved by molecular replacement with the PLP bound to the catalytic lysine. Further protein engineering will give rise to mutants with an expanded substrate scope. The main goal is to employ this enzyme in a cascade reaction where its function would be to decarboxylate hydroxy amino acids.

\section{References:}

1. R. Kourist et al., Engineering the promiscuous racemase activity of an arylmalonate decarboxylase. Chemistry 17, 557-563 (2011).

2. F. Jordan et al., Catalysis in Enzymatic Decarboxylations: Comparison of Selected Cofactordependent and Cofactor-independent Examples. ACS Catal 3, 1601-1617 (2013). 